\title{
17-allylamino-17-demethoxygeldanamycin and ritonavir inhibit renal cancer growth by inhibiting the expression of heat shock factor-1
}

\author{
AKINORI SATO, TAKAKO ASANO, KEIICHI ITO and TOMOHIKO ASANO \\ Department of Urology, National Defense Medical College, 3-2 Namiki, Tokorozawa, Saitama 359-8513, Japan
}

Received December 12, 2011; Accepted February 8, 2012

DOI: $10.3892 /$ ijo.2012.1419

\begin{abstract}
Our previous study showed that the combination of a histone deacetylase (HDAC) inhibitor and an HIV protease inhibitor is effective against renal cancer cells. Because HDAC inhibition disrupts the chaperon function of heat shock protein (HSP) 90, we hypothesized that the combination of 17-allylamino-17-demethoxygeldanamycin (17-AAG), an inhibitor of HSP90, and the HIV protease inhibitor ritonavir would also act against renal cancer. The combination of 17-AAG and ritonavir induced apoptosis and inhibited the proliferation of renal cancer cells effectively. It also suppressed the expression of cyclin-dependent kinase 4 and cyclin D1, leading to the accumulation of the cells in the sub-G1 fraction. The expression of HSPs 27, 70 and 90 was increased by 17-AAG alone but reduced by $17-\mathrm{AAG}$ combined with ritonavir. The combination decreased the expression of heat shock factor-1 (HSF-1), an HSP transcription factor, and this might be one of the mechanisms of the effect of the combination. We have also found that silencing of HSF-1 by siRNA inhibited the proliferation of renal cancer cells and that in surgically resected specimens the levels of HSF-1 expression in renal cancer tissue are higher than those in normal parenchyma. This is the first study showing the beneficial effect of combining 17-AAG and ritonavir and our data suggest that HSF-1 may be a novel therapeutic target in the treatment of renal cancer.
\end{abstract}

\section{Introduction}

Although recently introduced novel anticancer agents such as inhibitors of tyrosine kinase $(1,2)$ and the mammalian target of rapamycin (3) are promising against advanced renal cancer, new treatment approaches are needed because curative agents are not yet available.

Correspondence to: Dr Akinori Sato, Department of Urology, National Defense Medical College, 3-2 Namiki, Tokorozawa, Saitama 359-8513, Japan

E-mail: zenpaku@ndmc.ac.jp

Key words: renal cancer, heat shock factor-1, 17-allylamino-17demethoxygeldanamycin, ritonavir
Inhibition of heat shock protein 90 (HSP90) destabilizes the cancer cell's aberrant protein subset, leading to protein degradation by proteasomes (4). The geldanamycin derivative 17-allylamino-17-demethoxy-geldanamycin (17-AAG) is the first HSP90 inhibitor to be used clinically (5). It seems to be a promising anticancer agent with a novel mechanism of action, but a phase II clinical trial investigating the efficacy of 17-AAG found it not to be effective against renal cancer (6).

We have previously shown that the combination of the histone deacetylase inhibitor suberoylanilide hydroxamic acid (SAHA) and the HIV protease inhibitor ritonavir is effective against renal cancer by inhibiting the HDAC function and expression, leading to extensive histone acetylation (7). SAHA inhibits HDAC6, and ablation of HDAC6 has been shown to induce hyperacetylation of HSP 90, disrupting its chaperone function $(8,9)$. We therefore thought that combining ritonavir with 17-AAG would be more effective against renal cancer because 17-AAG could inhibit HSP90 function more directly than SAHA does.

In the present study, we investigated the combined effect of 17-AAG and ritonavir against renal cancer by using renal cancer cell lines and found that the combination inhibited the renal cancer growth by inhibiting the expression of heat shock factor-1 (HSF-1), an HSP transcriptional factor. We also inferred from RNAi study results and the finding of higher expression of HSF-1 in surgically resected specimens that the expression of HSF-1 is associated with cell proliferation.

\section{Materials and methods}

Cell culture. Renal cancer cells (A-498, ACHN, Caki-1) were obtained from the American Type Culture Collection (Rockville, MD, USA) and grown in DMEM and MEM media containing $10 \%$ fetal bovine serum and $0.3 \%$ penicillin-streptomycin (Invitrogen, Carlsbad, CA, USA).

Reagents. 17-AAG purchased from Merck (Darmstadt, Germany) and ritonavir purchased from Tronto Research Chemicals (North York, ON, Canada) were dissolved in dimethyl sulfoxide (DMSO) and stored at $-20^{\circ} \mathrm{C}$ until use.

Cell viability assay. Cells were plated in a 96-well culture plate and incubated in medium containing 17-AAG $(0-1 \mu \mathrm{M})$ with or without ritonavir $(0-50 \mu \mathrm{M})$ for $48 \mathrm{~h}$. Cell viability was assayed 
A

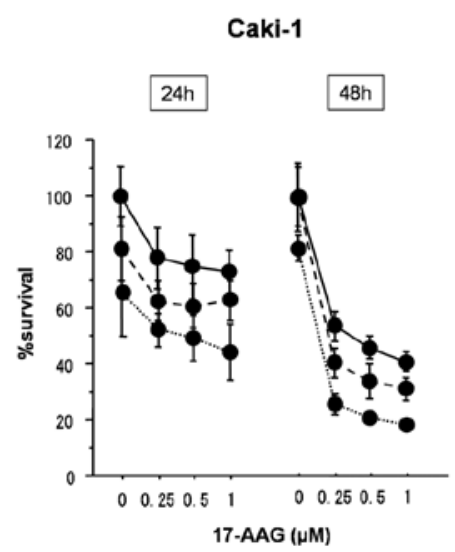

B

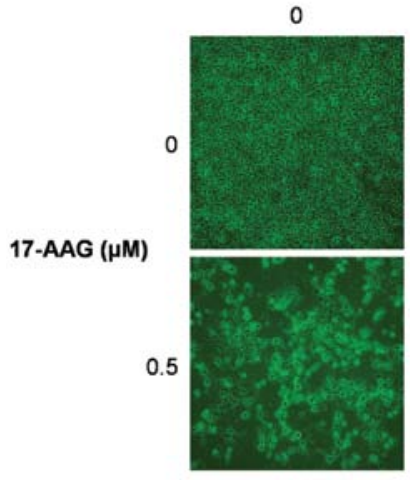

Ritonavir $(\mu \mathrm{M})$

25

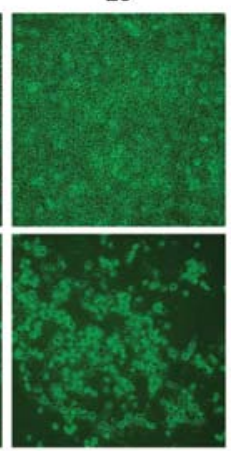

A-498

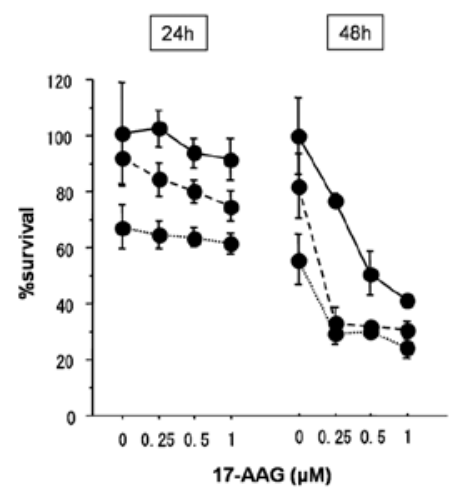

17-AAG ( $\mu \mathrm{M})$
ACHN

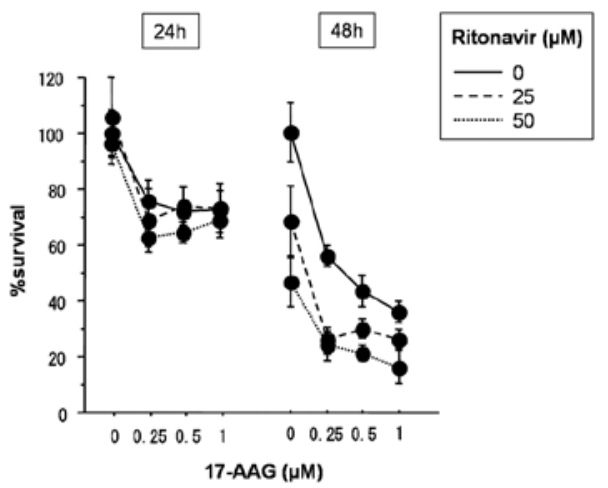

C
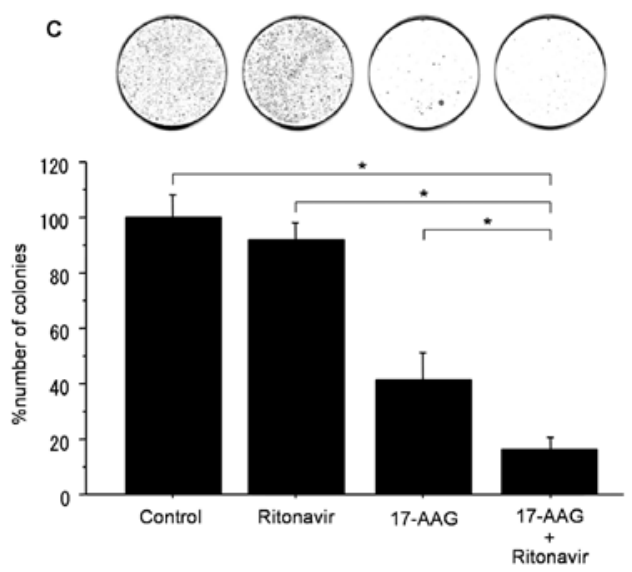

Figure 1. Combination of 17-AAG and ritonavir inhibits renal cancer growth. (A) Inhibition of cell proliferation by 48 -h treatment with $17-\mathrm{AAG}$ and ritonavir (MTS assay; $\mathrm{n}=6$, mean \pm SD). (B) Photomicrographs of Caki-1 cells treated with 17-AAG and ritonavir for $48 \mathrm{~h}$ (original magnification, $\mathrm{x} 200$ ). (C) Colony formation assay. Cells were treated for $24 \mathrm{~h}$ with either DMSO, $0.05 \mu \mathrm{M}$ 17-AAG, $50 \mu \mathrm{M}$ ritonavir, or both $0.05 \mu \mathrm{M} 17-\mathrm{AAG}$ and $50 \mu \mathrm{M}$ ritonavir and then incubated for 7 days. The combination of $17-\mathrm{AAG}$ and ritonavir inhibited colony formation significantly $\left(\mathrm{n}=3\right.$, mean $\left.\pm \mathrm{SD},{ }^{*} \mathrm{p}<0.05\right)$.

by the MTS assay (Promega, Madison, WI, USA) according to the manufacturer's protocol.

Colony formation assay. Caki-1 cells $\left(5 \times 10^{3}\right)$ were plated into $10-\mathrm{cm}$ plates one day before being cultured for $24 \mathrm{~h}$ in media containing $0.05 \mu \mathrm{M}$ 17-AAG and/or $50 \mu \mathrm{M}$ ritonavir. They were then given fresh media and allowed to grow for 1 week before they were fixed with $100 \%$ methanol, stained with Giemsa's solution, and counted.

Apoptosis assay. Forty-eight hours after the cell culture in media containing 17-AAG and/or ritonavir, the induction of apoptosis was evaluated by Annexin V assay, measuring the cells in the sub-G1 fraction, and changes in the expression of survivin, X-linked inhibitor of apoptosis protein (XIAP), active caspase 3 and cleaved poly(ADP-ribose) polymerase (PARP) by western blot analysis.

Flow cytometry. Cells were plated in a 6-well culture plate and treated under the indicated conditions for $48 \mathrm{~h}$. They were then washed with PBS and harvested by trypsinization. For cell cycle analysis, the harvested cells were resuspended in citrate buffer, stained with propidium iodide, and analyzed by flow cytometry. For Annexin V assay, the cells were stained with Annexin $\mathrm{V}$ according to the manufacturer's instruction (Beckman Coulter, Marseille, France) and then analyzed by flow cytometry. Data analysis was done by using CellQuest Pro Software (BD Biosciences, San Jose, CA, USA).

Western blot analysis. Equal amounts of protein from wholecell lysates were subjected to SDS-PAGE and transferred onto nitrocellulose membranes that were then probed with primary antibodies specific for anti-cyclin D1, anti-cyclin dependent kinase (CDK) 4, anti-HSF-1 (Santa Cruz Biotechnology, Santa Cruz, CA, USA), anti-actin (Millipore, Billerica, MA, USA), anti-cleaved PARP, anti-HSP90, anti-HSP70, anti-HSP27 (Cell Signaling Technology, Danvers, MA, USA), anti-survivin (Alpha Diagnostic International, San Antonio, TX, USA), anti-XIAP, anti-acetylated histone (Abcam, Cambridge, UK). This probing was followed by treatment with horseradish-tagged secondary antibodies (GE Healthcare, Piscataway, NJ, USA) and visualization with chemiluminescence solutions (GE Healthcare).

Silencing of HSF-1 by siRNA transfection. Caki-1 cells were plated in 6-well (for western blot analysis) or 96-well (for MTS assay) culture plates one day before being given $100 \mathrm{nM}$ 
A
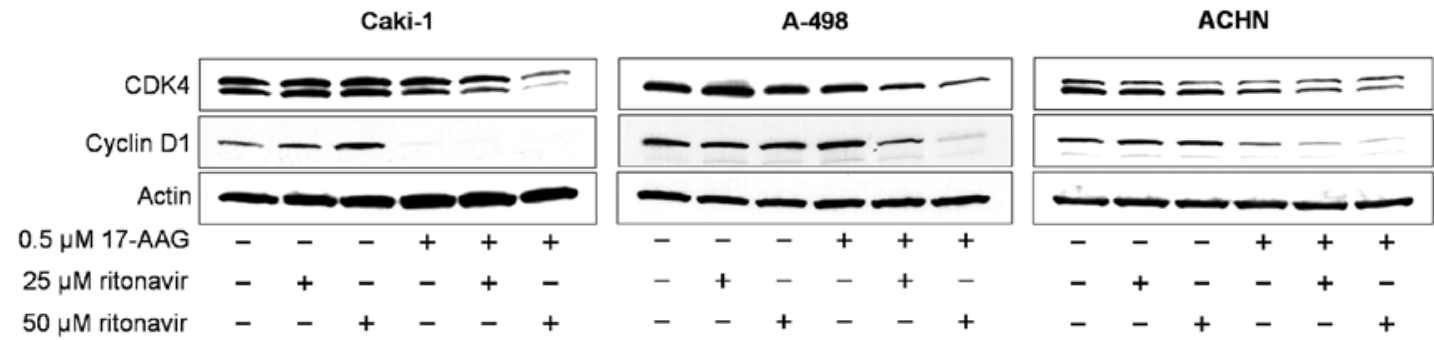

B
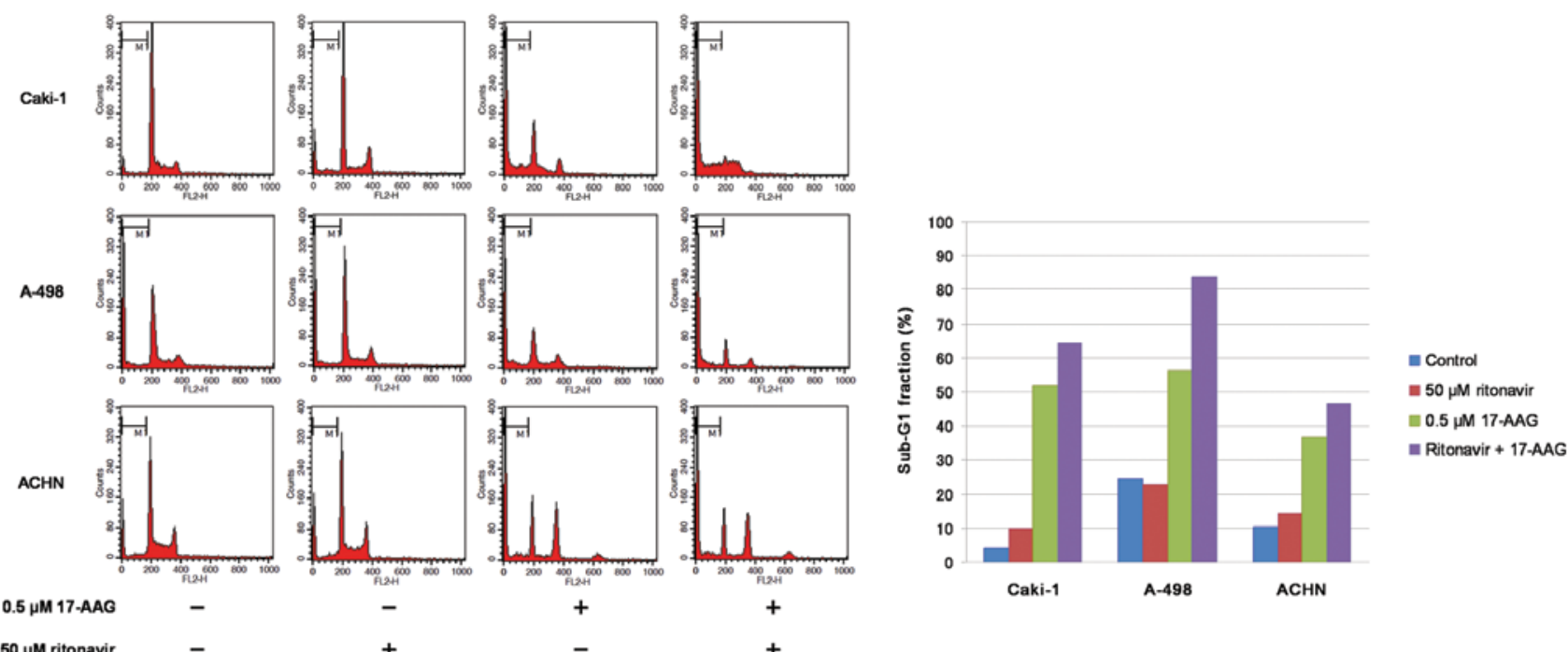

Figure 2. Changes in the cell cycle and the expression of cell-cycle-associated proteins after treatment with 17-AAG and ritonavir. (A) Western blotting for cyclin D1 and CDK4 $48 \mathrm{~h}$ after treatment with 17-AAG and ritonavir. The combination decreased the expression of cyclin D1 and CDK4. Actin served as a loading control. Representative blots are shown. (B) Cell cycle analysis $48 \mathrm{~h}$ after treatment with 17-AAG and ritonavir. The combination drastically increased the number of cells in the sub-G1 fraction. The graph shows the percentage of the cells that are in the sub-G1 fraction. Representative results of flow cytometry are shown.

HSF-1-specific siRNA or non-sense siRNA (Santa Cruz Biotechnology) that had been mixed with Lipofectamine 2000 (Invitrogen) according to the manufacturer's protocol. The cells were then cultured for $72 \mathrm{~h}$ and assayed for HSF-1 expression by western blot analysis and cell proliferation by MTS assay.

Expression of HSF-1 in surgically resected specimens. Renal cancer tissue and normal renal parenchyma obtained at renal cancer surgery performed at our department was stored at $-80^{\circ} \mathrm{C}$ until use. Whole-cell lysates were obtained from 32 specimens and subjected to western blot analysis. The level of HSF-1 expression was semiquantified using the public domain NIH Image program and normalized to the level of actin expression. Relative HSF-1 density was calculated by dividing the HSF-1 level in cancer tissue by the HSF-1 level in normal parenchyma.

Statistical analyses. The statistical significance of observed differences between samples was determined using the MannWhitney U test (StatView software, SAS Institute, Cary, NC, USA). Differences were considered significant at $\mathrm{p}<0.05$.

\section{Results}

Effect of combination of 17-AAG and ritonavir on cell proliferation. MTS assay showed that 48-h treatment with combinations of 17-AAG and ritonavir inhibited the proliferation of renal cancer cells effectively (Fig. 1A and B). The combined effect seen when $25 \mu \mathrm{M}$ ritonavir was given in combination seems to be synergistic. The combination of $17-\mathrm{AAG}$ and $50 \mu \mathrm{M}$ ritonavir also inhibited the clonogenecity of Caki-1 cells significantly $(\mathrm{p}<0.05)$ (Fig. 1C).

Changes in the cell cycle and the expression of cell cycleassociated proteins. To test the combined effect of 17-AAG and ritonavir on the cell cycle, we evaluated changes in the cell cycle and the expression of cell cycle-associated proteins. In Caki-1 and ACHN cells, $0.5 \mu \mathrm{M}$ 17-AAG decreased the expression of cyclin D1 but ritonavir alone did not change the expression of either cyclin D1 or CDK4 (Fig. 2A). These changes were compatible with the observed changes in the cell cycle; that is, 17-AAG increased the number of the cells in the sub-G1 fraction and ritonavir had only slight effects on the cell cycle. Interestingly, the combination of 17-AAG and ritonavir greatly 
A

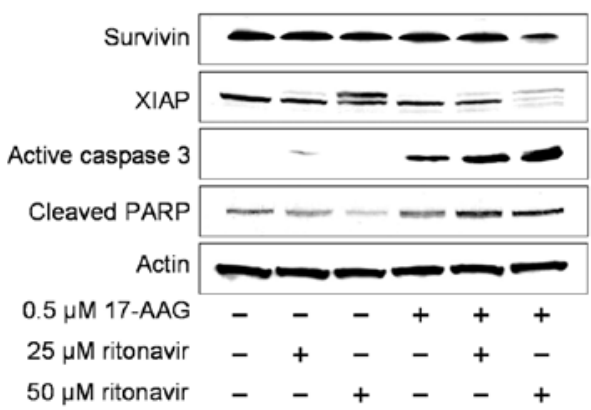

B

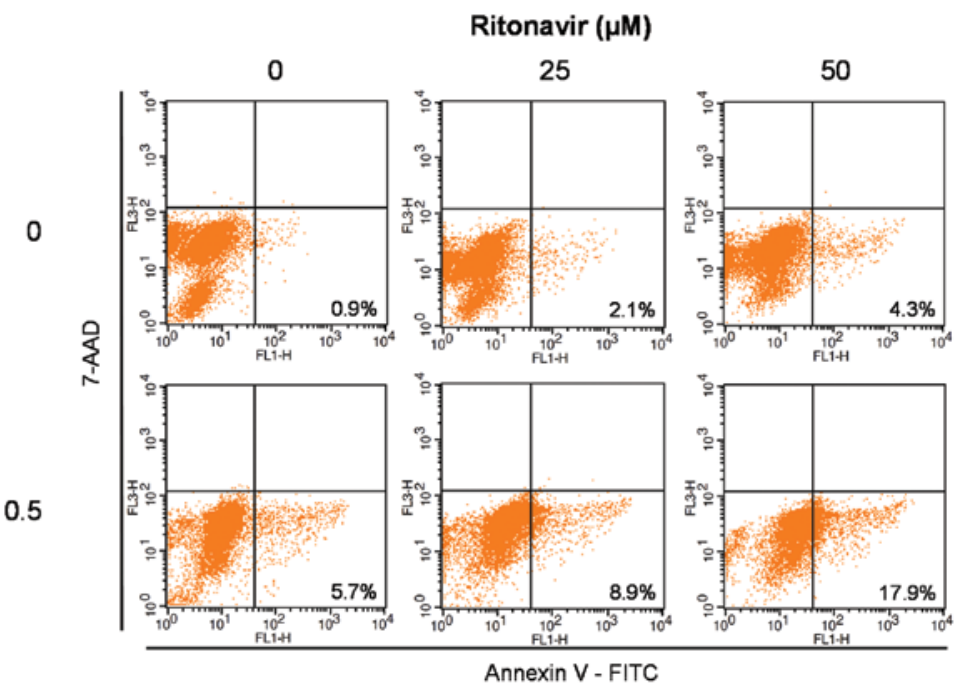

Figure 3. Induction of apoptosis by the combination of 17-AAG and ritonavir in Caki-1 cells. (A) Western blotting for survivin, XIAP, cleaved PARP, and active caspase 3 after 48-h treatment. The combination decreased the expression of survivin and XIAP, and it markedly increased the expression of cleaved PARP and active caspase 3. Actin served as a loading control. Representative blots are shown. (B) Annexin V assay. Caki-1 cells were stained with Annexin V $48 \mathrm{~h}$ after treatment under the indicated conditions and analyzed by flow cytometry. The combination increased the number of Annexin V-positive cells. The inset in each graph shows the percentage of the Annexin V-positive cells. Representative results of flow cytometry are shown.
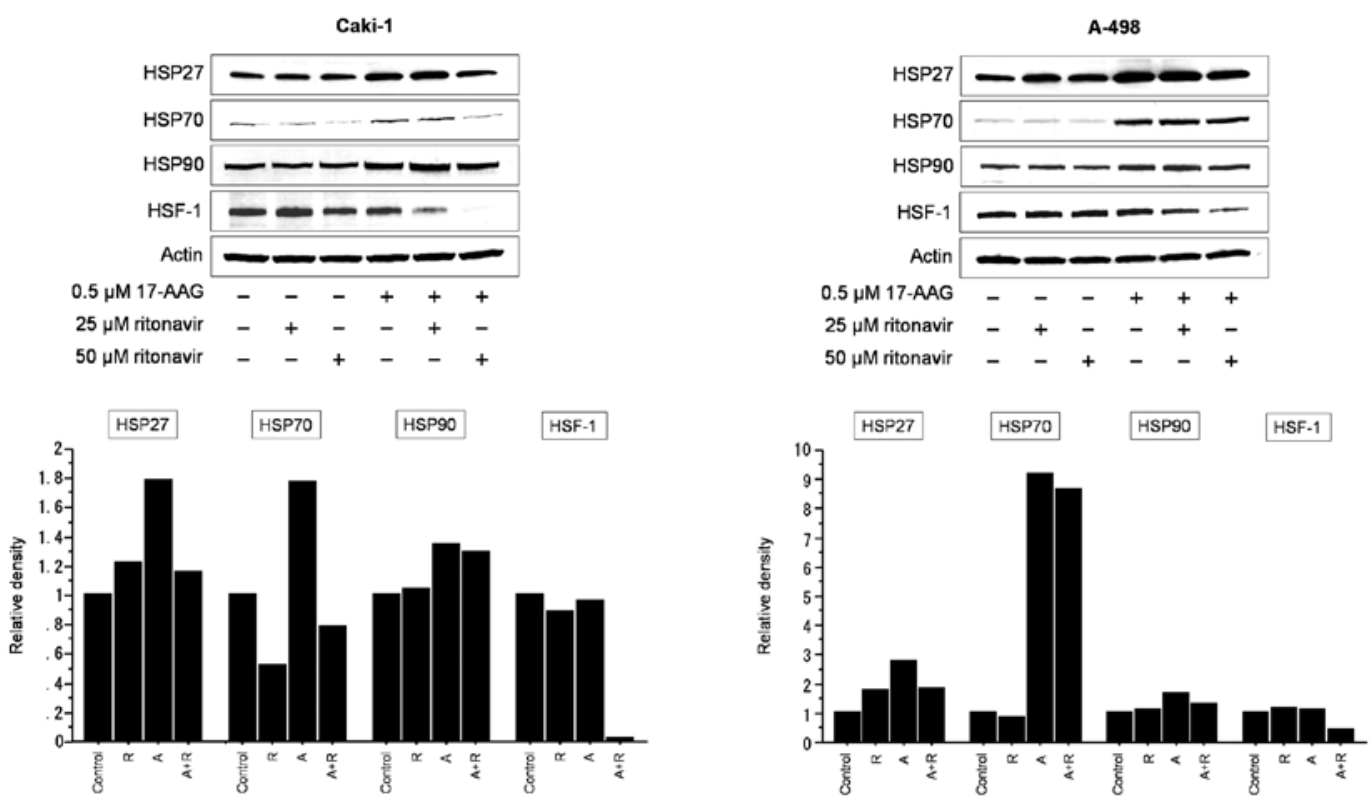

Figure 4. Combination of 17-AAG and ritonavir suppressed 17-AAG-induced HSP expression. Western blotting for HSPs and HSF-1 $48 \mathrm{~h}$ after treatment under the indicated conditions. 17-AAG alone increased the expression of HSPs, and the combination of 17-AAG and ritonavir suppressed this increase. Note that the expression of HSF-1 was decreased by the combination. Actin served as a loading control. Representative blots are shown. The graph shows relative density of HSPs (control =1). R, $50 \mu \mathrm{M}$ ritonavir; A, $0.5 \mu \mathrm{M}$ 17-AAG.

decreased the expression of both cyclin D1 and CDK4 in all the cell lines tested, leading to the accumulation of the cells in the sub-G1 fraction (Fig. 2B).

Induction of apoptosis by the combination of 17-AAG and ritonavir. To evaluate induction of apoptosis by the combination therapy, we first investigated the changes in the expression of antiapoptotic proteins, active caspase 3 , and cleaved PARP in Caki-1 cells. The combination therapy decreased the expression of survivin and XIAP and increased the expression of cleaved PARP and active caspase 3 (Fig. 3A). Flow cytometry also showed that the number of Annexin V-positive cells was increased synergistically by the combination of $17-\mathrm{AAG}$ and ritonavir (Fig. 3B).

17-AAG-induced HSP expression is suppressed by ritonavir in combination. 17-AAG inhibits HSP90 but it generally increases the expression of HSPs (10), which could attenuate the effect of 17-AAG. We therefore evaluated the HSP expression changes by the combination of 17-AAG and ritonavir (Fig. 4). In Caki-1 
A

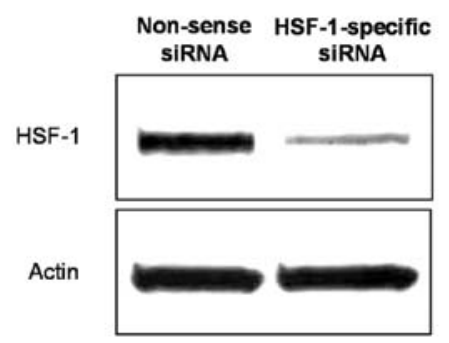

B

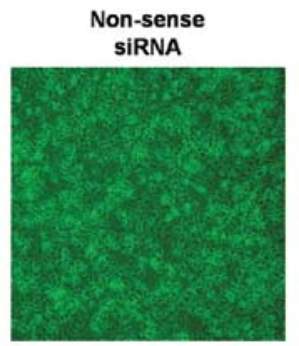

HSF-1-specific SiRNA

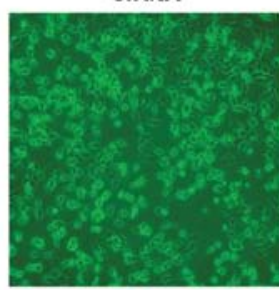

C

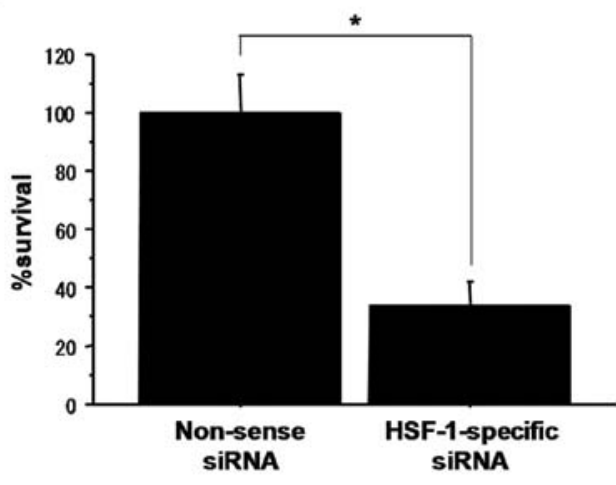

Figure 5. Suppression of HSF-1 expression by siRNA inhibited the proliferation of Caki-1 cells. (A) Western blotting. The transfection of HSF-1-specific siRNA for $72 \mathrm{~h}$ suppressed HSF-1 expression. Actin served as a loading control. Representative blots are shown. (B) Photomicrographs and (C) MTS assay ( $\mathrm{n}=6$, mean \pm SD). Suppression of HSF-1 expression by siRNA inhibited cell proliferation significantly. ${ }^{\mathrm{p}} \mathrm{p}=0.0039$.
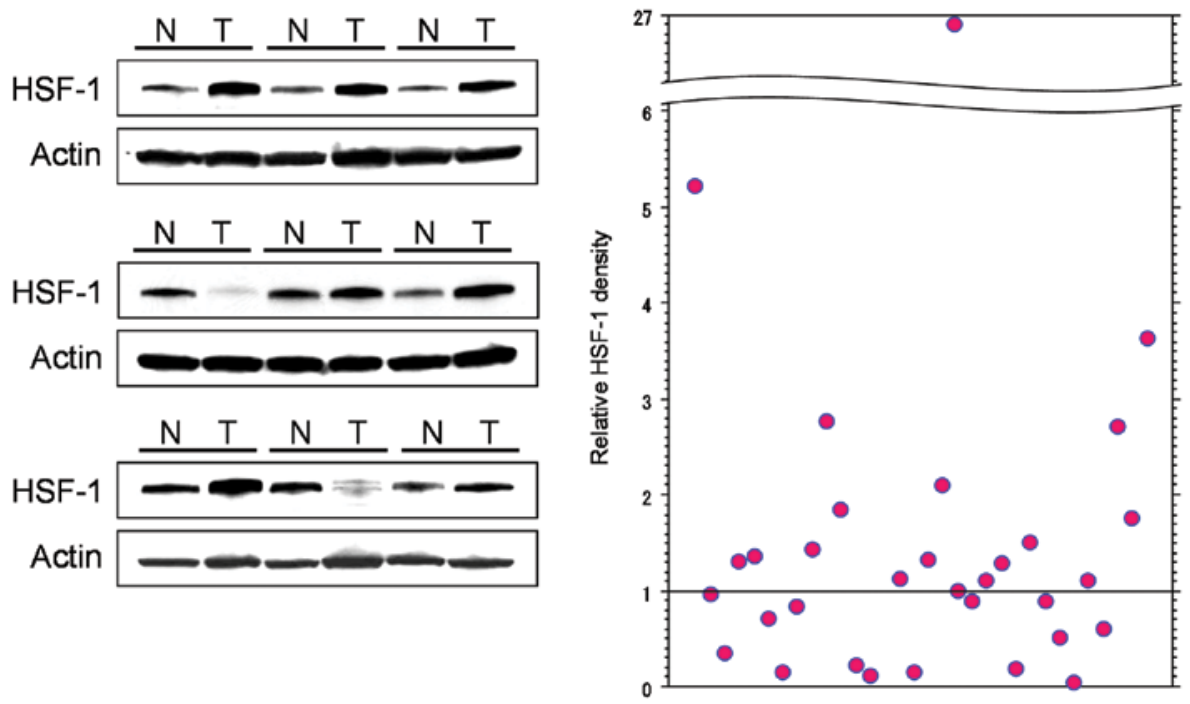

Figure 6. Expression of HSF-1 in surgically resected specimens. Left, examples of the western blotting for HSF-1 in renal cancer tissue (T) and normal renal parenchyma (N). Actin served as a loading control. Right, scattered plot of relative density of HSF-1 [(HSF-1 level in cancer tissue) / (HSF-1 level in normal parenchyma)].

and A-498 cells, $0.5 \mu \mathrm{M} 17-\mathrm{AAG}$ increased the expression of HSPs 27, 70, and 90. Interestingly, ritonavir in combination with 17-AAG suppressed this increase of the HSP expression. We also found that ritonavir in combination with 17-AAG decreased the expression of HSF-1, an HSP transcription factor, which in part explains the suppression of 17-AAG-induced expression of HSPs by ritonavir.

Effect of HSF-1 suppression by siRNA. The suppression of HSF-1 expression seems to be an important mechanism of action of the combination of 17-AAG and ritonavir. To investigate the association of HSF-1 with cell proliferation, we evaluated the effect of HSF-1 silencing in Caki-1 cells. The transfection of HSF-1-specific siRNA for $72 \mathrm{~h}$ suppressed HSF-1 expression and inhibited cell proliferation (Fig. 5A and B). MTS assay results also confirmed this significant antiproliferative effect of HSF-1 silencing (Fig. 5C). These results suggest that HSF-1 is associated with the proliferation of Caki-1 cells.

Expression of HSF-1 in surgically resected specimens. Because our in vitro experiment suggested the importance of HSF-1 expression in proliferation of renal cancer, we investigated the 
difference in the expression of HSF-1 between normal tissue and cancer tissue by using surgically resected specimens (Fig. 6, left). HSF-1 expression was higher (relative HSF-1 level $>1$ ) in cancer tissue in 18 of 33 patients (Fig 6, right), suggesting the importance of the HSF-1 expression in renal cancer.

\section{Discussion}

Inhibiting HSP90 is a novel and attractive approach to cancer treatment. 17-AAG is the first HSP90 inhibitor used clinically (5), but a clinical trial showed it not to be effective against renal cancer when administered alone (6). Ritonavir is an HIV protease inhibitor widely used for treating HIV infection and has recently been found to have antitumor activity. It has been shown to inhibit the Akt (11) and NF- $\kappa \mathrm{B}$ (12) signaling pathways, but the exact mechanism of its action is still unknown. We have recently shown that the combination of SAHA and ritonavir inhibits renal cancer growth synergistically by suppressing the expression and function of HDAC (7). Because inhibition of HDAC6 inhibits HSP90 function $(8,9)$, we thought that the combination of 17-AAG and ritonavir would also be effective against renal cancer because 17-AAG directly inhibits the function of HSP90.

The combination of 17-AAG and ritonavir inhibited the growth of renal cancer cells effectively, leading to apoptosis. One mechanism of action would be perturbation of the cell cycle. In all the cell lines tested, the combination decreased the expression of cyclin D1 and CDK4 and increased the number of cells in the sub-G1 fraction. Previous studies showed that 17-AAG induced cell cycle arrest and apoptosis by downregulating cyclin D1 and CDK4 $(10,13)$. Ritonavir was also reported to deplete CDKs 2, 4, and 6 and cyclin D1 (14). Furthermore, both 17-AAG and ritonavir were shown to inhibit the transcription factor $\mathrm{NF}-\kappa \mathrm{B}(12,15)$ and cyclin $\mathrm{D} 1$ is a target gene of $\mathrm{NF}-\kappa \mathrm{B}(16)$. The combination of $17-\mathrm{AAG}$ and ritonavir may thus act cooperatively to decrease the expression of cyclin D1 and CDK4 drastically, leading to apoptosis. Furthermore, in Caki-1 cells the combination decreased the expression of survivin and XIAP, which may be one mechanism of action causing apoptosis. Survivin and XIAP are both important antiapoptotic proteins in renal cancer. Survivin has been reported to be associated with the proliferation of renal cancer cells (17) and is a potential molecular target for treating renal cancer (18-20). On the other hand, XIAP has been shown to be a prognostic marker in renal cancer (21). The combination of 17-AAG and ritonavir is therefore attractive for treating renal cancer because it suppresses the expression of survivin and XIAP.

Although 17-AAG inhibits the function of HSP90, it generally increases the expression of HSPs (10), which could attenuate the effect of 17-AAG. Furthermore, in the present study the administration of 17-AAG alone increased the expression of HSPs 90, 70 and 27. The transcriptional regulation of different HSP genes is under the control of HSFs (22). The function of HSF-1 is regulated by HSP90 and inhibition of HSP90 releases HSF-1 from the HSP90 complex, which results in its activation and translocation to the nucleus (23). The combination suppressed the expression of HSF-1 and this would inhibit HSP induction by 17-AAG, enhancing 17-AAG activity. The exact mechanism of the regulation of HSF-1 expression, however, remains unknown. Further study is needed to clarify the mecha- nism of the regulation of HSF-1 expression and why ritonavir in combination decreased the HSF-1 expression.

Because HSP90 is required for the stability and function of a number of signaling proteins that promote the growth of cancer cells (24), the expression of its transcription factor HSF-1 is also thought to be associated with cancer growth. There are, however, few studies directly focusing on the association between HSF-1 expression and cancer growth. Cai et al showed that increased expression of HSFs might render colon carcinoma cells resistant to the chemopreventive effects of butyrate (25). According to our RNAi study, HSF-1 seems to play an important role in renal cancer growth. Furthermore, using surgically resected specimens, we have found that the expression of HSF-1 is higher in renal cancer tissue than in normal renal parenchyma. To our knowledge, this is the first study that showed HSF-1 may be a therapeutic target in the treatment of renal cancer. Targeting HSF-1 by, for example, using specific inhibitors of HSF-1 might also be effective against renal cancer.

In summary, the combination of 17-AAG and ritonavir inhibited renal cancer growth effectively and one mechanism of action might be the suppression of HSF-1 expression. HSF-1 may thus be a future novel therapeutic target in the treatment of renal cancer.

\section{References}

1. Ratain MJ,Eisen T, Stadler WM, et al: Phase II placebo-controlled randomized discontinuation trial of sorafenib in patients with metastatic renal cell carcinoma. J Clin Oncol 24: 2505-2512, 2006.

2. Escudier B, Eisen T, Stadler WM, et al: Sorafenib in advanced clear-cell renal-cell carcinoma. N Engl J Med 356: 125-134, 2007.

3. Motzer RJ, Escudier B, Oudard S, et al: Phase 3 trial of everolimus for metastatic renal cell carcinoma: final results and analysis of prognostic factors. Cancer 116: 4256-4265, 2010.

4. Sepp-Lorenzino L, Ma Z, Lebwohl DE, Vinitsky A and Rosen N: Herbimycin A induces the $20 \mathrm{~S}$ proteasome- and ubiquitindependent degradation of receptor tyrosine kinases. J Biol Chem 16580-16587, 1995.

5. Taldone T, Gozman A, Maharaj R and Chiosis G: Targeting Hsp90: small-molecule inhibitors and their clinical development. Curr Opin Pharmacol 8: 370-374, 2008

6. Ronnen EA, Kondagunta GV, Ishill N, et al: A phase II trial of 17-(Allylamino)-17-demethoxygeldanamycin in patients with papillary and clear cell renal cell carcinoma. Invest New Drugs 24: 543-546, 2006.

7. Sato A, Asano T, Horiguchi A, Ito K, Sumitomo M and Asano T: Combination of suberoylanilide hydroxamic acid and ritonavir is effective against renal cancer cells. Urology 76: 764.e7-13, 2010.

8. Bali P, Pranpat M, Bradner J, et al: Inhibition of histone deacetylase 6 acetylates and disrupts the chaperone function of heat shock protein 90: a novel basis for antileukemia activity of histone deacetylase inhibitors. J Biol Chem 280: 26729-26734, 2005.

9. Fiskus W, Ren Y, Mohapatra A, et al: Hydroxamic acid analogue histone deacetylase inhibitors attenuate estrogen receptor-alpha levels and transcriptional activity: a result of hyperacetylation and inhibition of chaperone function of heat shock protein 90 . Clin Cancer Res 13: 4882-4890, 2007.

10. Georgakis GV, Li Y and Younes A: The heat shock protein 90 inhibitor 17-AAG induces cell cycle arrest and apoptosis in mantle cell lymphoma cell lines by depleting cyclin D1, Akt, Bid and activating caspase 9. Br J Haematol 135: 68-71, 2006.

11. Kumar S, Bryant CS, Chamala S, et al: Ritonavir blocks AKT signaling, activates apoptosis and inhibits migration and invasion in ovarian cancer cells. Mol Cancer 8: 26, 2009.

12. Dewan MZ, Tomita M, Katano H, et al: An HIV protease inhibitor, ritonavir targets the nuclear factor-kappaB and inhibits the tumor growth and infiltration of EBV-positive lymphoblastoid B cells. Int J Cancer 124: 622-629, 2009. 
13. Georgakis GV, Li Y, Rassidakis GZ, Medeiros LJ and Younes A The HSP90 inhibitor 17-AAG synergizes with doxorubicin and U0126 in anaplastic large cell lymphoma irrespective of ALK expression. Exp Hematol 34: 1670-1679, 2006.

14. Srirangam A, Mitra R, Wang M, et al: Effects of HIV protease inhibitor ritonavir on Akt-regulated cell proliferation in breast cancer. Clin Cancer Res 12: 1883-1896, 2006.

15. Myung SJ, Yoon JH, Kim BH, Lee JH, Jung EU and Lee HS Heat shock protein 90 inhibitor induces apoptosis and attenuates activation of hepatic stellate cells. J Pharmacol Exp Ther 330: 276-282, 2009

16. Hinz M, Krappmann D, Eichten A, et al: NF-kappaB function in growth control: regulation of cyclin D1 expression and G0/ G1-to-S-phase transition. Mol Cell Biol 19: 2690-2698, 1999.

17. Sato A, Oya M, Ito K, et al: Survivin associates with cell proliferation in renal cancer cells: regulation of survivin expression by insulin-like growth factor-1, interferon-gamma and a novel NF-kappaB inhibitor. Int J Oncol 28: 841-846, 2006.

18. Sato A, Ito K, Asano T, Sumitomo M, Asano T and Hayakawa M: Synergistic effect of survivin-specific small interfering RNA and topotecan in renal cancer cells: topotecan enhances liposomemediated transfection by increasing cellular uptake. Int J Oncol 30: 695-700, 2007.

19. Sato A, Ito K, Asano T, Sumitomo M, Asano T and Hayakawa M: Topotecan and small interfering RNA suppress survivin expression synergistically in Caki-1 renal cancer cells: Direct suppression of survivin and enhancement of transfection efficiency by topotecan. Int J Oncol 32: 171-176, 2008.
20. Sato A, Asano T, Ito K, Sumitomo M, Asano T and Hayakawa M: A potential novel combination therapy targeting survivin in renal cancer cells: inhibition of survivin expression by topotecan and hexamethylene bisacetamide. Mol Med Rep 2: 423-428, 2009.

21. Ramp U, Krieg T, Caliskan E, et al: XIAP expression is an independent prognostic marker in clear-cell renal carcinomas. Hum Pathol 35: 1022-1028, 2004.

22. Pirkkala L, Nykanen P and Sistonen L: Roles of the heat shock transcription factor in regulation of the heat shock response and beyond. FASEB J 15: 1118-1131, 2001.

23. Zou J, Guo Y, Guettouche T, Smith DF and Voellmy R: Repression of heat shock transcription factor HSF1 activation by HSP90 (HSP90 complex) that forms a stress-sensitive complex with HSF1. Cell 94: 471-480, 1998.

24. Neckers L: Heat shock protein 90: the cancer chaperone. J Biosci 32: 517-530, 2007.

25. Cai J, Kirlin WG, Chen Y, Yan X, Jones DP and Sartorelli AC: Overexpression of heat shock factor 1 inhibits butyrate-induced differentiation in colon cancer cells. Cell Stress Chaperones 11: 199-207, 2006 